\title{
SOME RESULTS ON COMMON FIXED POINTS AND BEST APPROXIMATION
}

\author{
ABDUL RAHIM KHAN, ABDUL LATIF, ARJAMAND BANO \\ AND NAWAB HUSSAIN
}

\begin{abstract}
We prove a common fixed point result for $(f, g)$-nonexpansive maps and then derive certain results on best approximation. Our results generalize the results of Al-Thagafi [1], Jungck and Sessa [6], Khan, Hussain and Thaheem [8], Latif [9, 10] and Sahab, Khan and Sessa [12].
\end{abstract}

\section{Preliminaries.}

Let $X$ be a linear space. A $p$-norm on $X$ is a real valued function $\|\cdot\|_{p}$ on $X$ with $0<p \leq 1$, satisfying the following conditions:

(i) $\|x\|_{p} \geq 0$ and $\|x\|_{p}=0 \Leftrightarrow x=0$

(ii) $\|\lambda x\|_{p}=|\lambda|^{p}\|x\|_{p}$

(iii) $\|x+y\|_{p} \leq\|x\|_{p}+\|y\|_{p}$

for all $x, y \in X$ and all scalars $\lambda$. The pair $\left(X,\|\cdot\|_{p}\right)$ is called a $p$-normed space. It is a metric space with a translation-invariant metric $d_{p}$ defined by $d_{p}(x, y)=\|x-y\|_{p}$ for all $x, y \in X$. If $p=1$, we obtain the concept of a normed space. It is well known that the topology of every Hausdorff locally bounded topological linear space is given by some $p$-norm, $0<p \leq 1[9,10]$. The space $l_{p}$ and $L_{p}, 0<p \leq 1$ are $p$-normed spaces. A $p$-normed space is not necessarily a locally convex space.

Let $C$ be a subset of $p$-normed space $X$, and let $f, g$ be self maps of $X$. The set $C$ is called starshaped with respect to $q \in C$ if for all $x \in C$ and for all $k, 0 \leq k \leq 1$, $k x+(1-k) q \in C$. The set $C$ is said to be starshaped if it is starshaped with respect to one of its elements.

Each convex set is necessarily starshaped. For any $x_{0} \in X$, define $d_{p}\left(x_{0}, C\right)=$ $\inf _{u \in C}\left\|x_{0}-u\right\|_{p}, P_{C}\left(x_{0}\right)=\left\{y \in C:\left\|y-x_{0}\right\|_{p}=d_{p}\left(x_{0}, C\right)\right\}$ the set of best $C$ approximants to $x_{0}$ and $D_{C}^{f}\left(x_{0}\right)=\left\{y \in C: f(y) \in P_{C}\left(x_{0}\right)\right\}$. Note that $P_{C}\left(x_{0}\right)$ contains $f\left(D_{C}^{f}\left(x_{0}\right)\right)$ and $g\left(D_{C}^{g}\left(x_{0}\right)\right)$. Assume that $D_{C}^{f, g}\left(x_{0}\right)=D_{C}^{f}\left(x_{0}\right) \cap D_{C}^{g}\left(x_{0}\right)$ and $D=P_{C}\left(x_{0}\right) \cap D_{C}^{f, g}\left(x_{0}\right)$.

Received and revised July 21, 2003.

2000 Mathematics Subject Classification. 47H10, 54H25.

Key words and phrases. p-normed space, best approximation, common fixed point, commuting maps, property of contractiveness and joint continuity. 
Let $\Im=\left\{h_{\alpha}\right\}_{\alpha \in C}$ be a family of functions from $[0,1]$ into $C$ such that $h_{\alpha}(1)=\alpha$ for each $\alpha \in C$. The family $\Im$ is said to be contractive if there exists a function $\varphi:(0,1) \rightarrow$ $(0,1)$ such that for all $\alpha, \beta \in C$ and all $t \in(0,1)$ we have

$$
\left\|h_{\alpha}(t)-h_{\beta}(t)\right\|_{p} \leq[\varphi(t)]^{p}\|\alpha-\beta\|_{p}
$$

The family $\Im$ is said to be jointly continuous (resp. jointly weakly continous) if $t \rightarrow t_{0}$ in $[0,1]$ and $\alpha \rightarrow \alpha_{0}$ in $C$ (resp. $t \rightarrow t_{0}$ in $[0,1]$ and $\alpha \stackrel{w}{\rightarrow} a_{0}$ in $C$ ), then $h_{\alpha}(t) \rightarrow h_{\alpha_{0}}\left(t_{0}\right)$ (resp. $\left.h_{\alpha}(t) \stackrel{w}{\rightarrow} h_{\alpha_{0}}(t)\right)$ in $C$; here $\rightarrow$ and $\stackrel{w}{\rightarrow}$ denote the strong and weak convergence respectively.

It is known that if $C \subseteq X$ is $q$-starshaped and $h_{x}(t)=(1-t) q+t x,(x \in C ; t \in[0,1])$, then $\Im=\left\{h_{x}\right\}_{x \in C}$ is a contractive jointly continuous family with $\varphi(t)=t$. Thus the class of subsets of $X$ with the property of contractiveness and jointly continuity contains the class of starshaped sets which in turns contains set class of convex sets (see, [3] and [8]). If for a subset $C$ of $X$, there exists a contractive jointly continuous family of functions $\Im=\left\{h_{\alpha}\right\}_{\alpha \in C}$, then we say that $C$ has the property of contractiveness and joint continuity.

A map $T: X \rightarrow X$ is $(f, g)$-contraction [7,9], if there exists a real number $k \in(0,1)$ such that

$$
\left.\|T x-T y\|_{p} \leq k\|f x-g y\|\right) p \text { for all } x, y \in X .
$$

If in the above inequality $k=1$, then $T$ is called $(f, g)$-nonexpansive. Also if $f=g$, we say that $T$ is $f$-nonexpansive. We denote the boundary of $C$ by $\partial C$, closure of $C$ by $\operatorname{cl}(C)$ and the set of fixed points of $T$ by $F(T)$.

Using fixed point theory, Brosowski [2], Meinardus [11] have established some interesting results on invariant approximation in the setting of normed spaces. Jungck and Sessa [6] have also obtained some results in approximation theory in the setting of normed spaces. Their work has been extended, generalized and unified by many authors; for example, see $[4,5,6,8,12,13]$.

Recently, Latif [9] has obtained the following results on common fixed points and best approximation, which generalize and extend the recent work of Al-Thagafi [1], Sahab, Khan and Sessa [12] and Kaneko [7] etc.

Theorem 1.1. Let $X$ be a p-normed space and $C$ a closed subset of $X$ which is starshaped with respect to $q$. Let $f, g$ and $T$ be continuous self-maps of $C$ such that $T(C) \subset f(C) \cap g(C)$, T commutes with $f$ and $g$, and $q \in F(f) \cap F(g)$. If $c l(T(C))$ is compact, $f$ and $g$ are affine, and $T$ is $(f, g)$-nonexpansive, then $F(f) \cap F(g) \cap F(T) \neq \varnothing$.

Theorem 1.2. Let $f, g$ and $T$ be self-maps of a p-normed space $X$ such that $x_{0} \in$ $F(f) \cap F(g) \cap F(T)$ and $C \subset X$ with $T(\partial C \cap C) \subset C$. Let $T$ be a $(f, g)$-nonexpansive map and continuous on $D \cup\left\{x_{0}\right\}$ such that $c l(T(D))$ is compact, and let $f$ and $g$ be continuous, surjective, affine and commute with $T$ on $D$. If $D$ is closed and starshaped with respect to $q \in F(f) \cap F(g)$, then $P_{C}\left(x_{0}\right) \cap F(f) \cap F(g) \cap F(T) \neq \varnothing$.

We also recall the following common fixed point result due to Jungck and sessa [6]. 
Theorem 1.3. Let $f$ be a continuous self-map of a compact metric space $(X, d)$. If $f x \neq f y$ implies $d(f x, f y)<d(g x, h y)$ for some self-maps $g$ and $h$ of $X$, commuting with $f$, then $F(f) \cap F(g) \cap F(h)$ is singleton.

The aim of this paper is to extend Theorem 1.1 and 1.2 to a domain which is not necessarily starshaped. An approximation result for two maps when underlying best approximation set $D$ need not be compact, will be established. Our results extend the corresponding results of Jungck and Sessa [6], Khan, Hussain and Thaheem [8], Latif [9, 10], Sahab, Khan and Sessa [12], Habiniak [4] and Singh [13].

\section{Common Fixed Points and Best Approximations}

Our first result generalizes Theorem 1.1, in the sense that the underlying domain $C$ need not be starshaped.

Theorem 2.1. Let $f, g$ and $T$ be continuous self-maps on a subset $C$ of a p-normed space $X$. Suppose that $T$ is $(f, g)$-nonexpansive and commutes with $f$ and $g$ on $C$. Suppose that $C$ is compact and has a contractive jointly continuous family $\Im=\left\{h_{x}\right\}_{x \in C}$ such that $f\left(h_{x}(\alpha)\right)=h_{f(x)}(\alpha)$ and $g\left(h_{x}(\alpha)\right)=h_{g(x)}(\alpha)$ for all $x \in C$ and $\alpha \in[0,1]$. Then $F(f) \cap F(g) \cap F(T) \neq \varnothing$.

Proof. For $n \in N$, let $\lambda_{n}=n(n+1)^{-1}$. Define

$$
T_{n}(x)=h_{(T x)}\left(\lambda_{n}\right), \quad x \in C .
$$

Then each $T_{n}$ is a well-defined self-map of $C$. Since $f$ and $T$ commute on $C$, it follows from the property of $\Im$ that

$$
T_{n}(f x)=h_{T f(x)}\left(\lambda_{n}\right)=h_{f T(x)}\left(\lambda_{n}\right)=f\left(h_{T(x)}\left(\lambda_{n}\right)\right)=f T_{n}(x) \quad(x \in C) .
$$

Thus for each $n, T_{n}$ commutes with $f$. Similarly, for each $n, T_{n}$ commutes with $g$.

Moreover, since $T$ is $(f, g)$-nonexpansive, we get

$$
\begin{aligned}
\left\|T_{n} x-T_{n} y\right\|_{p} & =\left\|h_{T(x)}\left(\lambda_{n}\right)-h_{T(y)}\left(\lambda_{n}\right)\right\|_{p} \\
& \leq\left[\varphi\left(\lambda_{n}\right)\right]^{p}\|T(x)-T(y)\|_{p} \\
& \leq\left[\varphi\left(\lambda_{n}\right)\right]^{p}\|f x-g y\|_{p} \\
& <\|f x-g y\|_{p}
\end{aligned}
$$

Then it follows from Theorem 1.3 that there exists $x_{n} \in C$ such that $x_{n} \in F\left(T_{n}\right) \cap F(f) \cap$ $F(g)$ for each $n$. Thus, by the compactness of $C$ it follows that the sequence, $\left\{x_{n}\right\}$ has a subsequence $\left\{x_{n_{i}}\right\}$ which converges to some $z \in C$ and hence $T\left(x_{n_{i}}\right) \rightarrow T(z)$. The joint continuity of $\Im$ gives

$$
x_{n_{i}}=T_{n_{i}}\left(x_{n_{i}}\right)=h_{T\left(x_{n_{i}}\right)}\left(\lambda_{n_{i}}\right) \rightarrow h_{T(z)}(1)=T z
$$


and thus the uniqueness of the limit implies $T z=z$. Also, since $f x_{n_{i}}=x_{n_{i}}=g x_{n_{i}} \rightarrow z$ using the continuity of $f$ and $g$ and the uniqueness of the limit, we have $f(z)=g(z)=z$, which completes the proof.

Theorem 2.2. Let $f, g$ and $T$ be self-maps of a p-normed space $X$ such that $x_{0} \in$ $F(f) \cap F(g) \cap F(T)$ and $C \subset X$ with $T(\partial C \cap C) \subset C$. Let $T$ is $(f, g)$-nonexpansive on $D \cup\left\{x_{0}\right\}$ and let $f$ and $g$ be continuous, surjective and commute with $T$ on $D$. Suppose that $D$ is compact and has a contractive jointly continuous family $\Im=\left\{h_{x}\right\}_{x \in D}$ such that $f\left(h_{x}(\alpha)\right)=h_{f(x)}(\alpha)$ for all $x \in D$ and $\alpha \in[0,1]$. Then $P_{C}\left(x_{0}\right) \cap F(f) \cap F(g) \cap F(T) \neq \varnothing$.

Proof. Let $y \in D$. Then $\left\|y-x_{0}\right\|_{p}=d_{p}\left(x_{0}, C\right)$ since $y \in P_{C}\left(x_{0}\right)$. Note that for any $k \in(0,1)$

$$
\left\|k x_{0}+(1-k) y+x_{0}\right\|_{p}=(1-k)^{p}\left\|y-x_{0}\right\|_{p}<d_{p}\left(x_{0}, C\right)
$$

It follows that the line segment $\left\{k x_{0}+(1-k) y: 0<k<1\right\}$ and the set $C$ are disjoint. Thus $y$ is not in the interior of $C$ and so $y \in \partial C \cap C$. Since $T(\partial C \cap C) \subset C, T y$ must be in $C$. Also since $f y \in P_{C}\left(x_{0}\right), x_{0} \in F(T) \cap F(g)$ and $T$ is $(f, g)$-nonexpansive on $D \cup\left\{x_{0}\right\}$, we have

$$
\left\|T y-x_{0}\right\|_{p}=\left\|T y-T x_{0}\right\|_{p} \leq\left\|f y-g x_{0}\right\|_{p}=\left\|f y-x_{0}\right\|_{p}=d_{p}\left(x_{0}, C\right),
$$

and hence $T y \in P_{C}\left(x_{0}\right)$. Moreover, since $T$ commutes with $f$ on $D$ and $x_{0} \in F(g) \cap F(T)$, we have

$$
\left\|f T y-x_{0}\right\|_{p}=\left\|T f y-T x_{0}\right\|_{p} \leq\left\|f^{2} y-g x_{0}\right\|_{p}=\left\|f^{2} y-x_{0}\right\|=d_{p}\left(x_{0}, C\right)
$$

and similarly, since $T$ commutes with $g$ and $x_{0} \in F(f) \cap F(T)$, we get

$$
\left\|g T y-x_{0}\right\|_{p} \leq\left\|g^{2} y-x_{0}\right\|_{p}=d_{p}\left(x_{0}, C\right) .
$$

Thus $f T y$ and $g T y$ are in $P_{C}\left(x_{0}\right)$ and so $T y \in D_{C}^{f, g}\left(x_{0}\right)$. Thus the definition of $D$ implies that $T y \in D$. Consequently $T$ maps $D$ into $D$. For $n \in N$, define the maps $T_{n}$ from $D$ into $D$ as in the proof of Theorem 2.1. As in the proof of Theorem 2.1, there exists $x_{n} \in D$ such that $x_{n} \in F\left(T_{n}\right) \cap F(f) \cap F(g)$ for each $n$. In particular $x_{n}=f x_{n}=g x_{n}$, by compactness of $D,\left\{x_{n}\right\}$ has a subsequence $\left\{x_{n_{i}}\right\}$ which converges to $z \in D$ and hence $T\left(x_{n_{i}}\right) \rightarrow T z$. Now, we complete the proof as that of Theorem 2.1.

Now if we take $f=g$ in Theorem 2.2, then $D=P_{C}\left(x_{0}\right) \cap D_{C}^{f}\left(x_{0}\right)$ and thus we obtain the following result which generalizes Theorem 3.2 of Al-Thagafi [1] in the sense that the best approximation set $D$ in it need not be starshaped.

Corollary 2.3. Let $f, T$ be self-maps of a p-normed space $X$ such that $T(\partial C \cap C) \subset$ $C$. Suppose that $f$ and $T$ are commuting on $D=P_{C}\left(x_{0}\right) \cap D_{C}^{f}\left(x_{0}\right), f$ is continuous on $D, T$ is $f$-nonexpansive on $D \cup\left\{x_{0}\right\}$ and $f(D)=D$. Suppose that $D$ is compact and has a contractive jointly continuous family $\Im=\left\{h_{x}\right\}_{x \in D}$ such that $f\left(h_{x}(\alpha)\right)=h_{f(x)}(\alpha)$ for all $x \in D$ and all $\alpha \in[0,1]$. Then, $P_{C}\left(x_{0}\right) \cap F(f) \cap F(T) \neq \varnothing$. 
We observe that if $f\left(P_{C}\left(x_{0}\right)\right)=P_{C}\left(x_{0}\right)=g\left(P_{C}\left(x_{0}\right)\right)$, then $P_{C}\left(x_{0}\right)=D_{C}^{f, g}\left(x_{0}\right)$; consequently Theorem 2.2 is true for $D=P_{C}\left(x_{0}\right)$ and hence we have the following extension of the result due to Hicks and Humprise [5] and Sahab, Khan and Sessa [12].

Theorem 2.4. Let $f$ and $T$ be self maps on $X, x_{0} \in F(T) \cap F(f)$ and $C$ be a subset of $X$ such that $T(\partial C \cap C) \subset C$. Suppose that $f$ and $T$ are commuting on $D=P_{C}\left(x_{0}\right)$, $f$ is continuous on $D, T$ is $f$-nonexpansive on $D \cup\left\{x_{0}\right\}$ and $f(D)=D$. Suppose that $D$ is compact and has a contractive jointly continuous family $\Im=\left\{h_{x}\right\}_{x \in D}$ such that $f\left(h_{x}(\alpha)\right)=h_{f(x)}(\alpha)$ for all $x \in D$ and $\alpha \in[0,1]$. Then $P_{C}\left(x_{0}\right) \cap F(f) \cap F(T) \neq \varnothing$.

Recall that dual space $X^{*}$ (the dual of $X$ ) separates points of $X$ if for each nonzero $x \in X$, there exists $\phi \in X^{*}$ such that $\phi(x) \neq 0$. In this case the weak topology on $X$ is well-defined. Notice that if $X$ is not locally convex space, then $X^{*}$ need not separate the points of $X$. For example, if $X=L_{p}[0,1], 0<p<1$, then $X^{*}=\{0\}$. However there are some non-locally convex spaces (such as the $p$-normed spaces $L_{p}, 0<p<1$ ) whose dual separates the points.

In the following result we consider $X$ a complete $p$-normed space whose dual separates the points of $X$.

Theorem 2.5. Let $T$ and $f$ be selfmaps on $X, C$ a subset of $X$ such that $T(\partial C \cap C) \subset$ $C$ and $x_{0} \in F(T) \cap F(f)$. Let $D=P_{C}\left(x_{0}\right) \cap D_{C}^{f}\left(x_{0}\right)$ be a nonempty weakly compact set. Suppose that $f$ is continuous in the weak and the strong topologies on $D, f(D)=D$, and $T$ is $f$-nonexpansive map on $D \cup\left\{x_{0}\right\}$ which commutes with $f$ on $D$. If $D$ has a contractive family of functions $\Im=\left\{h_{x}\right\}_{x \in D}$ such that $f\left(h_{x}(\alpha)\right)=h_{f(x)}(\alpha)$ for all $x \in D$ and all $\alpha \in[0,1]$, then $f$ and $T$ have a common fixed point in $D$ provided either (i) $T$ is weakly continuous and family $\Im$ is jointly weak continuous or (ii) $T$ is completely continous and $\Im$ is jointly continuous.

Proof. As in the proof of Theorem 2.2, $T$ maps $D$ into $D$. Define the maps $T_{n}$ as in Theorem 2.2. Then $T_{n}$ commutes with $f$ and $T_{n}(D) \subseteq D=f(D)$. Since the family $\Im$ is contractive and $T$ is $f$-nonexpansive we have

$$
\left\|T_{n} x-T_{n} y\right\|_{p} \leq\left[\phi\left(\lambda_{n}\right)\right]^{p}\|T x-T y\|_{p} \leq\left[\phi\left(\lambda_{n}\right)\right]^{p}\|f x-f y\|_{p} \quad \text { for all } x, y \in D .
$$

So, by Theorem 5 [6], there exists a unique $x_{n} \in D$ such that $x_{n}=T_{n} x_{n}=f x_{n}$ for each $n$. Since $D$ is weakly compact, there is a subsequence $\left\{x_{n_{j}}\right\}$ of $\left\{x_{n}\right\}$ converging weakly to some $p \in D$. But, $f$ is weakly continuous so we have $f p=p$. Now if (i) holds, then $T x_{n_{j}} \stackrel{w}{\rightarrow} T p$ and hence $x_{n_{j}}=h_{T\left(x_{n_{j}}\right)}\left(\lambda_{n_{j}}\right) \stackrel{w}{\rightarrow} h_{T(p)}(1)=T(p)$. Also since $x_{n_{j}} \stackrel{w}{\rightarrow} p$ and the weak topology is Hausdorff, we get $T p=p$. Now suppose the condition (ii) holds. Since $x_{n_{j}} \stackrel{w}{\rightarrow} p$ and $T$ is completely continuous, we have $T x_{n_{j}} \rightarrow T p$. Now using the joint continuity of $\Im$ we get

$$
x_{n_{j}}=h_{T\left(x_{n_{j}}\right)}\left(\lambda_{n_{j}}\right) \rightarrow h_{T(p)}(1)=T(p) .
$$

Thus $T x_{n_{j}} \rightarrow T^{2} p$ and consequently $T z=z$ where $z=T p$. But

$$
f z=f T p=T f p=T p=z,
$$


which completes the proof.

\section{References}

[1] M. A. Al-Thagafi, Common fixed points and best approximation, J. Approx. Theory 85 (1996), 318-323.

[2] B. Brosowski, Fixpunktsatze in der approximations theorie, Mathematicae (Cluj) 11(1969), 195-220.

[3] W. G. Dotson Jr., On fixed points of nonexpansive mappings in nonconvex sets, Proc. Amer. Math. Soc. 38 (1973), 155-156.

[4] L. Habiniak, Fixed point theorems and invariant approximation, J. Approx. Theory 56 (1989), 241-244.

[5] T. L. Hicks and M. D. Humphries, A note on fixed point theorems, J. Approx. Theory 34(1982), 221-225.

[6] G. Jungck and S. Sessa, Fixed point theorems in best approximation theory, Math. Japon. 42(1995), 249-252.

[7] H. Kaneko, Single-valued and multivalued f-contraction, Boll. U.M.I. 6 (1985), 29-33.

[8] A. R. Khan, N. Hussain and A. B. Thaheem, Applications of fixed point theorems to invariant approximation, Approx. Theory and Appl. 16(2000), 48-55.

[9] A. Latif, Common fixed points versus best approximation, Tamkang J. Math. 32(2001), 1-5.

[10] A. Latif, A result on best approximation in p-normed spaces, Arch. Math. 37(2001), 71-75.

[11] G. Meinardus, Invarianze bei Linearen approximation, Arch. Rational Mech. Anal. 14 (1963), 301-303.

[12] S. A. Sahab, M. S. Khan and S. Sessa, A result in best approximation theory, J. Approx. Theory 55(1988), 349-351.

[13] S. P. Singh, An application of fixed point theorem to approximation theory, J. Approx Theory 25(1979), 89-90.

Department of Mathematical Sciences, King Fahd University of Petroleum and Minerals, Dhahran-31261, Saudi Arabia.

E-mail: arahim@kfupm.edu.sa

Department of Mathematics, King Abdul Aziz University, P.O.Box 80203, Jeddah 21589, Saudi Arabia.

E-mail: Latifmath@yahoo.com

Department of Mathematics, Gomal University, D. I. Khan, Rakistan.

E-mail: arjamandbano2002@yahoo.com

Department of Mathematics, King Abdul Aziz University, P.O.Box 80203, Jeddah 21589, Saudi Arabia.

E-mail: mnawab2000@yahoo.com 